\title{
Stem Cell Reviews and Reports: Adult Stem Cells and Tissue Regeneration Section
}

\author{
Edward W. Scott ${ }^{1}$
}

Published online: 20 February 2017

(C) Springer Science+Business Media New York 2017

Hematopoietic Stem Cells (HSC) are the adult stem cells that started the field of intensive research on Stem Cell Biology. In the past decade or so many additional adult stem cells (ASC) have been described in a wide array of tissues and organs. Comparative Biology approaches have shown many convergent biological properties that define "Stemness" in adult tissues. HSC were defined by their clonal ability to restore homeostasis to the blood system following ablative transplantation. There is no natural law that states all adult stem cells will be capable of similar feats in similar transplantation studies. Hence the robust debates about stem versus progenitor status for various ASC populations.

SCRR's newly organized section on Adult Stem Cells and Tissue Regeneration seeks to publish rigorous primary studies on the biology and activities of ASC within the context of the normal function of their parent tissues/organs, as well as ASC response to: injury, transplantation, exogenous engineering and manipulation etc. Comparative approaches to better define common ASC traits and the key tissue specific differences in function are also encouraged. In addition, we invite reviews on emerging areas of ASC biology. Reviews that contextualize contradictory evidence in the field with the goal of enucleating new studies to resolve outstanding gaps in ASC knowledge are highly encouraged.
Adult Stem cells responsible for the normal maintenance and repair/regeneration of their respective tissues/ organs. Advances in understanding the genetic and biologic pathways involved in normal tissue maintenance lend credence to developing clinically efficacious cell based therapies for human disease. These approaches include everything from direct ASC transplantation to engineered scaffolds and 3D printing of replacement tissue structures. This section of SCCR will also feature studies designed to enhance the native functionality of resident ASC.

In summary, the overarching goal of the Adult Stem Cells and Tissue Regeneration section of Stem Cell Reviews and Reports is to publish primary scientific studies on the function and use of ASC, and thought provoking review articles that synthesize current advances in stem cell biology, tissue engineering and organogenesis to further the development of therapies that enhance tissue repair and regeneration for the treatment a wide array of human diseases.

We are excited to hear about your opinion on the reorganized structure of SCRR and look forward to receiving your high-quality contributions to the new section in our journal.
Edward W. Scott

escott@ufl.edu

1 Department of Molecular Genetics and Microbiology, College of Medicine, University of Florida, Gainesville, FL, USA 